\title{
Claude Reichler et les échelles du paysage
}

\section{Michel Collot}

\section{OpenEdition}

Journals

Édition électronique

URL : http://journals.openedition.org/edl/832

DOI : $10.4000 /$ edl. 832

ISSN : 2296-5084

\section{Éditeur}

Université de Lausanne

\section{Édition imprimée}

Date de publication : 15 mai 2015

Pagination : 143-162

ISBN : 978-2-940331-45-1

ISSN : 0014-2026

\section{Référence électronique}

Michel Collot, "Claude Reichler et les échelles du paysage », Études de lettres [En ligne], 1-2 | 2015, mis en ligne le 15 mai 2018, consulté le 20 décembre 2020. URL : http://journals.openedition.org/edl/832 ; DOI : https://doi.org/10.4000/edl.832 


\section{CLAUDE REICHLER ET LES ÉCHELLES DU PAYSAGE}

A bien des égards, Claude Reichler apparaît comme un des pionniers d'une nouvelle démarche critique qui s'est récemment développée: la géographie littéraire, qui a trouvé notamment un terrain d'élection dans les récits de voyage et un thème privilégié dans les représentations du paysage. Sensible aux multiples enjeux de ce thème, Claude Reichler en propose une approche très complète et nuancée, qui fait la part aussi bien à la dimension physique du paysage, à ses valeurs historiques, sociales et culturelles qu'à l'expérience sensible de l'individu. Attentif à la façon dont ces représentations s'inscrivent dans le style même des œuvres qu'il étudie et dans leur iconographie, Claude Reichler se montre ainsi capable de parcourir à la fois les diverses échelles du texte et celles du paysage.

Le titre donné à ce colloque, "Les échelles du texte", propose à notre réflexion une métaphore à ma connaissance inédite dans le domaine de la théorie littéraire. L'expression n'est guère usitée, semble-t-il, que dans un sens très technique, désignant les variations auxquelles on peut soumettre la taille d'un document pour l'adapter à son format d'impression. Il s'agit plus précisément d'une métaphore spatiale, qui vient s'ajouter à la longue liste de celles qui, depuis la rhétorique antique, servent à décrire des faits linguistiques ou stylistiques, telles que plan, champ sémantique, niveaux de langue ou de lecture. Tout se passe comme si nous ne pouvions penser le langage qu'en le spatialisant, peut-être parce qu'il porte lui-même l'empreinte de notre expérience de l'espace. Gérard Genette faisait déjà remarquer que "le terme même de "métaphore spatiale" est presque un pléonasme, car les métaphores sont généralement tirées du lexique de l'étendue» ${ }^{1}$.

I. G. Genette, «Espace et langage», p. 107. 


\section{Géographie}

Cependant une des originalités de cette nouvelle métaphore, c'est qu'elle est empruntée au vocabulaire technique de la cartographie. Or, dans le dialogue entre la littérature et les sciences humaines, la géographie a longtemps fait figure de parent pauvre: dans les années 1960 et 1970, les études littéraires se référaient plutôt à la linguistique, à la sociologie ou à l'histoire. Mais depuis quelques décennies, les sciences de l'homme et de la société se sont montrées de plus en plus attentives à l'inscription des faits humains et sociaux dans l'espace. Ce «tournant spatial» a fait de la géographie une discipline de référence, comme en témoignent des néologismes comme "géohistoire», "géophilologie» ou "géophilosophie». Et les études littéraires ont suivi une évolution parallèle, qui a conduit à l'émergence de courants critiques baptisés "géopoétique», "géocritique» ou "géographie littéraire».

Ce dialogue avec la géographie apparaît très tôt dans le travail de Claude Reichler, comme en témoigne sa participation active à la Revue de géographie alpine, dont il a dirigé plusieurs numéros. Il en a été un des artisans les plus constants et les plus importants dans le monde universitaire francophone; il a notamment dirigé pendant cinq ans un programme de recherche sur "Le Paysage: perceptions, représentations, théories " ${ }^{2}$, et plusieurs thèses situées au croisement des deux disciplines, notamment celles de Samuel Thévoz et de Filippo Zanghi, au jury desquelles j'ai eu le plaisir de siéger récemment.

Du côté de la France, une enquête sur les thèses soutenues ou déposées depuis 1990 dans le domaine de la littérature française ou francophone $\mathrm{du} \mathrm{XX}^{\mathrm{e}}$ siècle a révélé qu'un nombre significatif d'entre elles portaient sur le cadre géographique de la production littéraire ou sur les représentations de l'espace dans les œuvres étudiées ${ }^{3}$. Beaucoup de colloques ont été consacrés ces dernières années à "L'inspiration géographique» 4 . Plusieurs formations de recherche s'attachent à l'étude de l'espace en littérature: citons, entre autres, les centres de recherche sur la littérature de

2. Projet IRIS 4, Nature, sciences, société (Universités de Lausanne et de Genève, EPFL).

3. Voir M. Collot, «Petit discours sur les méthodes», p. 41.

4. C'était le titre d'un colloque qui s'est tenu à Angers en 2003, et dont les actes ont été recueillis dans Le génie du lieu, sous la direction d'A. Bouloumié et d'I. TrivisaniMoreau. 
voyage (Paris IV / Clermont II), et sur les nouveaux espaces littéraires (Paris XIII), l'équipe d'accueil «Espaces humains et interactions culturelles» (Limoges), et le programme que je dirige à Paris III avec Julien Knebusch, "Vers une géographie littéraire " 5 . Et de nombreux ouvrages et numéros de revue ont accordé une attention particulière au paysage littéraire ${ }^{6}$.

Cet intérêt récent de la critique pour la représentation de l'espace dans les textes ne fait que répondre à un certain état de la pratique littéraire elle-même, qui fait aujourd'hui une large place à la dimension géographique. Après la vogue du textualisme et du formalisme, qui a dominé en France les années 1960 et 1970, s'est manifesté à partir des années 1980 le désir de rouvrir la littérature au monde. L'engouement pour les récits de voyage, qui concerne aussi bien la production et l'édition que les études littéraires, témoigne de cette reconquête de l'espace, illustrée notamment par le titre de l'ouvrage emblématique de Nicolas Bouvier, L'usage du monde, auquel Claude Reichler a consacré un bel article dans la revue Europe, en $2010^{7}$. Le récit de voyage, longtemps tenu en marge de la "grande littérature», est désormais reconnu comme un genre littéraire à part entière, et Claude Reichler a beaucoup contribué à cette réhabilitation: il a notamment fait paraître une importante anthologie sur le voyage dans les Alpes ${ }^{8}$ et il dirige aux Presses de l'Université ParisSorbonne la collection qui porte le même titre et où il a fait paraître récemment les Ecrits sur les Alpes de Ruskin; et il est toujours un membre actif du Centre de recherche sur la littérature des voyages?.

Cet élargissement de notre horizon littéraire a été favorisé par la montée en puissance des littératures francophones qui expriment une diversité de cultures et de territoires ${ }^{10}$. Dès 1993, Edouard Glissant

5. Pour plus d'informations sur ce programme, on peut se reporter à son «carnet de recherche" à l'adresse: http://geographielitteraire.hypotheses.org.

6. Voir notamment "Ecrire le paysage», numéro de la Revue des sciences humaines; M. Collot, Paysage et poésie; G. Sangirardi (dir.), Le paysage dans la littérature italienne; A. Gendrat-Claudel, Le paysage, "fenêtre ouverte" sur le roman.

7. C. Reichler, «Paysage et expérience de l'espace dans L'usage du monde».

8. C. Reichler (dir.), Le voyage dans les Alpes.

9. Il a été créé à Paris IV en 1984 par François Moureau et il est désormais dirigé à Clermont II par Philippe Antoine. On peut consulter son site internet à l'adresse: http://www.crlv.org.

Io. Voir M. Collot, A. Rodriguez (dir.), Paysage et poésies francophones. 
proclamait son ambition d'embrasser le "Tout-monde» ${ }^{11}$ et plus récemment un manifeste en faveur d'une "Littérature-monde» a été signé par vingt-sept écrivains désireux de faire sortir la littérature de langue française du cadre étroit de l'Hexagone ${ }^{12}$. En contrepoint de cette dimension planétaire s'est aussi développée une littérature ancrée dans un lieu ou une région. Elle traduit sans doute pour une part la résistance de nos sociétés à une mondialisation uniformisatrice qui met en péril les identités locales. Il me semble significatif que l'intérêt de Claude Reichler pour la littérature viatique se soit focalisée sur un territoire, celui des Alpes, qui, aussi vaste et transfrontalier soit-il, est tout de même lié fortement à l'identité spécifique, géographique et culturelle, de la Suisse.

On assiste aussi à la résurgence d'une littérature qu'on pourrait presque qualifier de régionaliste, si le terme n'était pas porteur de connotations dépassées. Beaucoup d'écrivains de ma génération, tels Pierre Michon, Pierre Bergounioux, ou Richard Millet, ont reçu «la province en héritage» mais, tout en assumant cet héritage, ils l'ont transformé pour exprimer une réalité qui a profondément bouleversé l'économie, la société, mais aussi la culture françaises au cours de la seconde moitié du XXe siècle, celle du déclin de la ruralité: "En même temps qu'elle entraîne la pensée vers les profondeurs abyssales de la mémoire", écrit Sylviane Coyault, "la province donne à voir le crépuscule d'un état de la civilisation ${ }^{13}$. Parallèlement, ce sont aussi les mutations de la ville, et notamment l'expansion et l'explosion des banlieues, qui servent non seulement de toile de fond mais de sujet à beaucoup de romans contemporains ${ }^{14}$, comme ceux qu'a étudiés dans sa thèse Filippo Zanghi.

Cette importance croissante d'une thématique géographique est inséparable de l'évolution récente des formes et des genres littéraires, comme l'avait suggéré dès 1945 Joseph Frank ${ }^{15}$. La poésie, sortant du cadre imposé par la versification régulière, explore l'espace de la page dans tous les sens et dans toutes ses dimensions, induisant un nouveau type de lecture, tabulaire et non linéaire. Mais cette spatialisation concerne aussi

\footnotetext{
II. Voir E. Glissant, Tout-monde et Traité du Tout-monde.

I2. M. Le Bris, J. Rouaud (dir.), Pour une littérature-monde.

I3. S. Coyault, La province en héritage, quatrième de couverture.

I4. Voir à ce propos A. Bergé, "Le tournant paysager de la littérature contemporaine».

I5. J. Frank, «La forme spatiale dans la littérature européenne».
} 
le récit: dans le Nouveau roman, tout se passe comme si la crise de la psychologie et de la chronologie avait conduit à une promotion de la dimension spatiale; au point qu'un de ses chefs de file, Michel Butor, a fini par abandonner la forme romanesque au profit d'ouvrages qui explorent de plus en plus largement l'espace de la planète et celui de la page et du livre, placés sous le signe du "Génie du lieu " ${ }^{16}$. Beaucoup de romans contemporains se présentent comme des "récits d'espace», comme ceux de Le Clézio, qui voit dans Le livre des fuites " une tentative pour découvrir l'espace» ${ }^{17}$, ou ceux de Jean Echenoz, dont le premier livre s'intitulait Le méridien de Greenwich, et qui dit écrire «des romans géographiques" ${ }^{18}$.

Or à cet investissement de la dimension spatiale par les littéraires et par les écrivains, correspond un intérêt croissant de certains géographes pour la littérature. Il s'inscrit dans le courant en faveur d'une "géographie humaniste», qui s'est développé depuis les années 1970 en réaction contre l'évolution d'une discipline qui, à la faveur du perfectionnement des moyens techniques, mathématiques et informatiques mis à sa disposition, avait tendance à privilégier une analyse objective et abstraite de l'espace au détriment de sa dimension humaine et sensible. Il y a dans cette approche "une visée phénoménologique destinée à remettre le sujet, le sens et les valeurs, un peu laissés pour compte, au cœur de la géographie» ${ }^{19}$. Elle s'est développée d'abord à partir de 1960 dans les pays anglo-saxons, privilégiant le lieu concret et vécu (place) plutôt que l'espace abstrait (space) ${ }^{20}$. Elle s'est affirmée en France dans les années 1970 : l'espace géographique est alors reconnu comme un espace perçu, un «espace vécu» ${ }^{21}$. Il n'est donc pas étonnant que les représentants de ce courant se soient souvent tournés vers la littérature pour y trouver l'expression de la dimension humaine et subjective des phénomènes géographiques; et que, réciproquement, un littéraire comme Claude Reichler ait collaboré à un collectif sur la géographie humaniste ${ }^{22}$.

16. M. Butor, Le génie du lieu (t. I) ; Où (t. II) ; Boomerang (t. III); Transit A / Transit B (t. IV); Le génie du lieu, Cinquième et dernier, autrement dit Gyroscope (t. V).

I7. Cf. P. Lhoste, «Je fuis l'Europe des esclaves», entretien avec Le Clézio.

I8. Voir notamment J. Echenoz, Je m'en vais.

19. M. Brosseau, Des romans-géographes, p. 18.

20. Y.-F. Tuan, Espace et lieu.

2I. Voir notamment A. Frémont, La région, espace vécu.

22. A. Bailly, R. Scariati (dir.), L’humanisme en géographie. 
Cette géographie n'est pas seulement humaine ou humaniste, mais culturelle, comme en témoigne le titre d'un de ses lieux d'expression, la revue Géographie et cultures. L'environnement humain est une construction culturelle autant qu'économique, et la littérature contribue à cette construction. Des thèses de géographie ont été soutenues portant sur un corpus exclusivement littéraire, comme celle de Marc Brosseau ${ }^{23}$ : consacrée au roman contemporain, celle-ci témoigne du souci de respecter la spécificité de la littérature, et d'être attentif à ce qu'elle peut apporter à la géographie. Selon Marc Brosseau, il y a une "pensée spatiale du roman", qui est indissociable de la forme littéraire elle-même; «l'étude de l'espace romanesque attire l'attention du géographe sur certains aspects négligés de l'espace et des lieux» et, en retour, "le point de vue géographique permet aussi de mettre en valeur des aspects négligés des rapports multiples [du roman] à l'espace» ${ }^{24}$.

\section{Paysage}

On assiste donc à une convergence remarquable entre les deux disciplines, les géographes trouvant dans la littérature la meilleure expression de la relation concrète, affective et symbolique qui unit l'homme aux lieux, et les littéraires se montrant de leur côté de plus en plus attentifs à l'espace où se déploie l'écriture. Un des terrains d'entente qui a favorisé leur rapprochement est sans nul doute la notion de "paysage», qui a connu de part et d'autre une promotion spectaculaire dans le dernier quart du siècle dernier. Elle présente en effet l'avantage de réunir toutes les dimensions de la "spatialité» humaine, entendue comme expérience et expression de notre rapport à l'espace.

Bien que nous l'abordions d'un point de vue et à propos de corpus différents, elle est aussi un point commun entre mes recherches dans le domaine de la poétique et de la théorie littéraire et celles de Claude Reichler, qui l'a placée depuis une vingtaine d'années au cœur de son travail d'historien de la culture et de la littérature. Nous en avons discuté

23. M. Brosseau, Des romans-géographes, Université Paris IV, 1992; publiée sous le même titre.

24. M. Brosseau, Des romans-géographes, p. 224. 
souvent au cours de colloques ou à distance, en échangeant nos livres; je me réjouis de l'occasion qui m'est donnée ici de poursuivre ce dialogue.

Avant d'examiner plus précisément l'usage qu'on peut faire de cette notion en littérature, je voudrais d'abord évoquer sa dimension anthropologique, qui a toujours été essentielle dans les travaux de Claude Reichler. Sa réflexion sur le paysage ne se limite ni à son expression littéraire ni à ses représentations picturales, ni même à l'histoire de la culture puisqu'elle débouche sur sa dimension sociale, économique et politique, comme en témoigne son rôle dans le Programme national de recherche sur les paysages des Alpes, qui portait en sous-titre: «de la représentation à l'action ", bel exemple d'application d'une recherche fondamentale aux pratiques contemporaines.

Le paysage est un carrefour où se rencontrent des éléments qui relèvent de la nature et de la culture, de la géographie et de l'histoire, de l'individu et de la société, du réel et du symbolique. De ces multiples dimensions seule une approche pluridisciplinaire peut saisir la spécificité et les interactions. C'est un phénomène complexe, qu'on peut étudier de multiples points de vue; chacun de nous, en fonction de sa spécialité et de sa compétence, est conduit à privilégier un de ces points de vue, mais il ne doit pas pour autant perdre de vue les autres. Le paysage, comme le texte, se prête à plusieurs niveaux de lecture qu'il faut éviter d'isoler les uns des autres. Claude Reichler se montre soucieux de parcourir ces diverses «échelles» du paysage ${ }^{25}$.

Cette ouverture aux multiples aspects du paysage s'oppose à une attitude qui a parfois prévalu dans ce qu'on appelle la théorie française du paysage. Elle a été un moment dominée par la thèse de l'"artialisation", terme emprunté par Alain Roger à Charles Lalo, qui l'avait trouvé luimême chez Montaigne. Selon cette thèse, le paysage ne serait perçu comme tel dans notre civilisation que par la médiation des artistes qui, en le représentant, ont imposé une certaine façon de voir le monde. Les tenants de l'artialisation réagissaient ainsi contre l'attitude naturaliste, en faisant du paysage un pur produit de la culture: selon le mot célèbre d'Oscar Wilde, c'est la nature qui imite l'art et non l'inverse.

Une telle alternative entre naturalisme et culturalisme méconnaît l'ambiguïté féconde du terme même de paysage qui, dans notre langue et

25. C. Reichler, La découverte des Alpes et la question du paysage (abrégé par la suite en $D A$, suivi de la pagination de l'extrait cité), p. 239. 
dans beaucoup d'autres, désigne à la fois un site (plus ou moins naturel) et sa représentation (culturelle). Et elle ne tient pas compte du troisième terme qui intervient dans sa définition, qui est le point de vue d'un sujet. Le paysage est un lieu où la nature et la culture se rencontrent aux yeux d'un homme. C'est à la fois un site, un regard et une image. C'est de cette triangulation que les études littéraires comme les sciences humaines et sociales doivent tenir compte; Claude Reichler montre bien, à propos de Saussure, que dans l'expérience du paysage "se nouent des rapports complexes entre la nature, la culture et le sujet percevant» $(D A, 60)$. Et il résume ainsi sa position, qui se rapproche de celle d'Augustin Berque:

Le paysage est composé de trois "échelles» ou de trois dimensions: la dimension bio-physique (objective), la dimension socio-culturelle et la dimension subjective individuelle. Elles sont en relation les unes avec les autres, selon des "trajets", des interactions et des tensions qui donnent à chaque fois un sens particulier au paysage. $(D A, 239)$

La recherche collective menée sur "les processus de perception" des paysages alpins a, quant à elle, abouti à l'élaboration d'un modèle encore plus complexe, qui situe le paysage au croisement de quatre instances: nature et culture, individu et société. Plus que l'identification de ces quatre pôles compte à mes yeux le repérage de leurs interactions, qui permet aux auteurs de la synthèse finale de dégager six dimensions fondamentales du paysage: corporelle et sensorielle, esthétique, identitaire, politique, économique et écologique ${ }^{26}$.

Le paysage apparaît ainsi, à la façon du texte selon Barthes, comme une sorte de "feuilleté" dont chaque couche se prête elle-même à diverses approches. Chacune de ses composantes naturelles peut faire en effet l'objet d'une étude spécialisée, faisant appel à la géologie, à la géomorphologie, à la climatologie, à la botanique, à l'écologie, ou à la zoologie... De telles enquêtes, aujourd'hui réservées aux sciences de la nature, ne sont pas absentes des textes qu'étudie Claude Reichler, souvent antérieurs au partage entre le discours scientifique et le discours littéraire: ainsi chez Saussure, il «constate que l'attention géologique et morphologique la plus minutieuse alterne avec de vives et belles descriptions dès que le voyageur présente à ses lecteurs des paysages. Plus que

26. Voir N. Backhaus, C. Reichler, M. Stremlow, Paysages des Alpes. 
d'alternance, il s'agit d'une complémentarité entre l'attitude esthétique et le travail scientifique» $(D A, 60)$.

Il y a dans le travail de Claude Reichler une visée encyclopédique; il est en cela fidèle à l'esprit des Lumières, mais aussi aux exigences du paysage, qui appelle, pour être compris dans sa complexité, un dépassement des savoirs spécialisés au profit d'une pensée globale, de ce que j'appelle une "pensée-paysage " ${ }^{27}$. Pour rendre compte de sa dimension socio-culturelle, il faudrait le concours de toutes les sciences humaines et sociales: économie, agronomie, sociologie, ethnologie, urbanisme, architecture, sciences politiques, histoire et notamment histoire des religions, des arts et de la littérature, la liste n'est pas exhaustive... Quant à ses résonances subjectives et individuelles, elles semblent d'un abord plus délicat, voire aléatoire; elles sont pourtant essentielles, car il n'y a de paysage à proprement parler que par et pour un sujet. L'étude de son rôle apparaît même comme stratégique. Si le paysage résulte de l'interaction entre un site, sa perception et sa représentation, il semble intéressant de partir du terme médian et médiateur: la perception d'un paysage doit tout autant à la configuration des lieux qu'aux figures de l'art et de la culture, et elle fait intervenir aussi les dispositions d'un sujet: sensations, souvenirs, sentiments, émotions, elle mobilise toute une "échelle des affects» $(D A, 79)$.

Pour ma part, je l'aborde à partir d'une phénoménologie de la perception, de l'imaginaire et des émotions. Claude Reichler part plutôt quant à lui de l'histoire culturelle, mais il sait faire dans sa démarche une place non négligeable à une phénoménologie de l'expérience paysagère. Il a même pu soutenir qu'au "niveau le plus fondamental» $(D A, 61)$ de celle-ci «interviennent des phénomènes qui appartiennent au corps du sujet et qui suspendent les données culturelles » $(D A, 74)$. L'historien de la culture doit dès lors «faire abstraction de l'effet des modèles visuels préformés dans le moment de la perception, pour comprendre celle-ci comme telle et éviter de la réduire à une reconnaissance» $(D A, 78)$.

Claude Reichler a pu ainsi montrer que l'expression littéraire du paysage échappait au moins en partie au modèle pictural du tableau, auquel on la ramène souvent. Les récits de voyage nous donnent à voir des paysages qui, loin de rester sages comme des images, sont en mouvement permanent, n'étant pas perçus d'un point de vue fixe mais lui-même

27. Voir M. Collot, La pensée-paysage. 
mobile. Le voyageur n'est pas toujours en face d'eux dans l'attitude du spectateur les contemplant à distance, il s'y trouve engagé: il est dedans, non devant. Claude Reichler insiste, avec Merleau-Ponty, sur l'aspect physique de cet engagement: «le paysage est un événement qui arrive au corps" $(D A, 78)$, dont il mobilise tous les sens et pas seulement la vue. Cette expérience polysensorielle «déplace les limites que lui assignent les modèles culturels et les cadres conceptuels dans lesquels elle se représente» $(D A, 61)$. Toujours neuve et singulière, elle permet de renouveler notre vision du paysage, sans pour autant s'abstraire du contexte dans lequel elle s'inscrit, "car la conscience n'est pas [...] solipsiste; elle est évidemment plongée dans une culture" $(D A, 78)$. De ce fait, "la théorie du paysage la plus adéquate combinerait» selon Claude Reichler "une phénoménologie et une histoire culturelle» $(D A, 78$ sq.). Ces deux approches, qu'on oppose souvent, sont en fait complémentaires; en les associant, Claude Reichler est parvenu à une conception très équilibrée du paysage, de ses composantes naturelles et culturelles, et de ses enjeux, individuels et collectifs:

Aventure d'une perception singulière et parcours d'un corps, acte d'échange entre l'homme et la nature, marque de l'appropriation qu'un sujet tente du monde, - le paysage est aussi une forme partagée par d'autres hommes, il constitue le réemploi (souvent inconscient) de modèles élaborés dans la culture; dans cette mesure il est une nature construite, identifiable, parlante. $(D A, 39)$

\section{Littérature}

Reste à savoir comment une telle conception peut éclairer l'expression littéraire du paysage. Il n'est pas évident de parler de "paysage» à propos de littérature: contrairement à ce qui se passe en peinture, il n'existe pas de genre littéraire qui corresponde à cette notion et la remise en cause de la mimesis par l'art et la littérature moderne a écarté de leurs préoccupations le paysage conçu comme une représentation du monde extérieur. Dans les années 1960 et 1970, en France notamment, l'influence du formalisme et du structuralisme avait abouti à une définition de la littérarité centrée sur la forme et la clôture du texte dont il convenait de faire une lecture strictement immanente. Se proposer d'étudier le paysage 
en littérature semblait relever alors d'un contresens ou d'une idéologie dépassée.

Il est vrai que les premières tentatives en faveur d'une géographie littéraire, dans la première moitié du $\mathrm{XX}^{\mathrm{e}}$ siècle, avaient surtout consisté à repérer dans les textes des référents géographiques sans trop se soucier du sens et de la forme qu'ils y revêtaient. Le premier en France à avoir essayé de donner à la géographie littéraire ses contours et ses méthodes est André Ferré, auteur d'une thèse sur la "géographie de Marcel Proust » 28 , et coéditeur avec Pierre Clarac de la première édition de La Recherche parue en 1954 dans la Bibliothèque de la Pléiade. Ce n'est pas un hasard si c'est un proustien, car La Recherche explore autant l'espace que le temps, la mémoire étant liée aux lieux, qui sont d'après le narrateur «les gisements profonds de (notre) sol mental ${ }^{29}$. La "géographie littéraire» d'André Ferré «se fonde sur ce très général postulat: qu'il existe nécessairement des relations entre toute œuvre humaine et le milieu terrestre où elle se localise, et que même dans ses aspects les plus spirituels et les plus rares, l'activité des hommes ne peut pas ne pas exprimer des relations de cette nature ${ }^{30}$.

Mais cet environnement reste un hors-texte, qui entretient avec le texte une relation d'extériorité, saisie en dehors de lui, dans la biographie de l'écrivain notamment. Les travaux d'André Ferré s'attachent principalement à recenser les lieux où a vécu ou qu'a connus un écrivain et à les comparer avec ceux qui sont évoqués dans son œuvre. Il en résume les résultats sur des «cartes biographiques" qui sont à la géographie littéraire ce que les chronologies sont à l'histoire littéraire. Même si André Ferré insiste sur l'écart entre les lieux de vie et les lieux de l'œuvre, ses recherches n'en subordonnent pas moins la géographie littéraire à une géographie référentielle, comme l'histoire littéraire a tendance à inféoder l'œuvre à la vie:

On cherche dans la biographie les données géographiques que laisse transparaître l'œuvre, celles qui peuvent permettre d'identifier et de localiser le site inspirateur. [...] Presque aucune ouvre littéraire ne

28. A. Ferré, Géographie de Marcel Proust (abrégé par la suite en GMP, suivi de la pagination des extraits cités).

29. M. Proust, Du côté de chez Swann, p. 182.

30. A. Ferré, Géographie littéraire, p. 10. 
manque de refléter, serait-ce tout indirectement, les circonstances de lieu de l'existence de l'écrivain ${ }^{31}$.

On retrouve ici les limites propres à toute théorie du reflet. Une géographie de la littérature ainsi conçue montre bien comment une œuvre s'ancre dans un territoire, mais elle oublie de montrer comment elle le transforme pour construire son propre espace, qui est celui de l'imaginaire et de l'écriture, qu'on ne trouve que dans le texte, et qu'on ne peut reporter sur aucune carte du monde connu. André Ferré le savait bien, lui qui, dans sa thèse, avait tenté d'«assigner aux divers paysages évoqués par Marcel Proust un emplacement sur la carte» (GMP, 85), mais avait dû reconnaître que la plupart des lieux de La Recherche "défi(ent) toute localisation unique et trop précise» $(G M P, 102)$, car «la géographie proustienne est une géographie toute psychologique, et même toute subjective et impressionniste» (GMP, 20).

Une géographie véritablement littéraire devrait intégrer cette dimension subjective et imaginaire, qui fait du lieu décrit un paysage. Cela n'exclut pas que celui-ci se fonde sur une géographie bien réelle et qu'il puisse être utile de la reconstituer voire de la cartographier. C'est le cas notamment pour les récits de voyage, qui suivent un itinéraire dont il importe d'identifier les étapes et les points de passage; c'est ce que fait Samuel Thévoz, qui annexe à sa thèse sur Bacot des cartes retraçant ses divers voyages au Tibet ${ }^{32}$. Commentant la lettre XXII d'Oberman, Claude Reichler met ses pas dans ceux du promeneur qui parcourt la forêt de Fontainebleau et il n'hésite pas à se reporter à une carte de l'époque pour vérifier l'exactitude des renseignements que Senancour donne sur les lieux, la végétation, les roches, les vues et qui font de cette lettre un véritable " guide» ${ }^{33}$.

Une telle démarche rend bien compte de la fonction documentaire d'un récit de voyage mais non de sa valeur littéraire. Dès lors qu'on s'intéresse à celle-ci, on entre dans un autre espace, qui n'est plus celui de la carte mais celui du paysage, qui est façonné à la fois par le point de vue d'un sujet et par la manière dont il s'exprime:

3I. Ibid., p. 24.

32. S. Thévoz, Un horizon infini.

33. C. Reichler, "Le marcheur romantique et la phénoménologie du chemin», p. 42. 
Il y a donc une relation symbolique intense entre l'espace, le texte [...] et le sujet. Les considérations purement territoriales et objectales ne suffisent pas à comprendre cela. Elles représentent une schématisation linéaire, une réduction à l'extériorité des parcours effectués par un sujet. Le récit de voyage littéraire, lui, montre une troisième dimension, celle de la configuration subjectale du monde vécu ${ }^{34}$.

"Le monde, le texte et le sujet prennent forme ensemble " ${ }^{35}$ au sein d'un paysage qui est à la fois extérieur, intérieur et littéraire. L'écriture est l'instrument d'une "co-naissance au monde et de soi-même", "les deux côtés de l'événement paysager (le subjectif et l'objectif) se plaçant dans un rapport de réciprocité, se donnant l'un à l'autre» ${ }^{36}$.

Reichler fait ici appel à une "compréhension phénoménologique" pour dénouer les fils de cet entrelacs qui se noue entre le subjectif et l'objectif. Une phénoménologie de la perception montre comment le regard permet au sujet à la fois de s'ouvrir au monde et de l'intérioriser. Chez Bouvier, «les changements d'échelle» qui, dans la cartographie, garantissent la proportion entre un territoire et l'image qui le représente objectivement, en "assurent» au contraire «l'appropriation subjective» ${ }^{37}$. Ils confèrent un rythme singulier aux descriptions de Bouvier qui donnent à voir le paysage selon des "échelles variables " ${ }^{38}$, télescopant le proche et le lointain, juxtaposant les détails aux vues d'ensemble.

Le style de l'écrivain informe le paysage autant que son regard, et une étude véritablement littéraire ne peut donc faire l'économie de son inscription textuelle et de sa dimension formelle. Dans L'usage $d u$ monde, note Reichler, "le style et le regard de l'auteur, sa manière propre d'écrire et de cadrer le monde, modèlent l'expérience et l'écriture et leur confèrent un rythme essentiellement discontinu, qui prend la forme de plages textuelles, de moments isolés attachés page à page " 39 . L'examen des manuscrits montre que le livre a été composé à partir de feuillets détachés analogues aux feuillets d'album où figurent les dessins de Vernet qui illustrent l'ouvrage, leur "écriture pictographique» faisant écho aux "propriétés visuelles» du texte. Ce rapprochement entre

34. C. Reichler, «Frontières du monde et territorialité du sujet», p. 129.

35. Ibid., p. 125.

36. C. Reichler, «Paysage et expérience de l'espace dans L'usage du monde», p. 140.

37. Ibid., p. 141.

38. Ibid., p. 137.

39. Ibid., p. 135. 
écriture et iconographie du paysage joue un rôle important dans les travaux de Claude Reichler et jusque dans son intérêt pour la vidéo; il appellerait tout un développement que je n'entreprendrai pas ici, faute de place, et pour centrer mon propos sur la dimension proprement littéraire du paysage, trop souvent envisagée à partir d'un modèle pictural, alors qu'elle relève moins de l'image que de l'imaginaire.

J'ai moi-même mis l'accent sur le motif de l'horizon ${ }^{40}$, intimement lié, dans notre civilisation, à la représentation du paysage, dont il illustre exemplairement la dimension subjective: c'est une ligne imaginaire, qu'on ne trouve reportée sur aucune carte, puisque son tracé varie en fonction du point de vue d'un sujet. Le choix de ce motif m'a été inspiré par mon corpus d'étude, qui est celui de la poésie moderne; or celleci est devenue principalement voire exclusivement lyrique et, en rejetant les formes traditionnelles, elle s'est efforcée aussi de mettre entre parenthèses les représentations qui informent notre vision du monde. Cela m'a conduit à voir surtout dans le paysage l'expression d'un imaginaire personnel; mais l'étude d'un autre corpus m'inciterait sans doute à y associer plus étroitement, à l'exemple de Claude Reichler, l'héritage culturel.

Cette remarque m'invite à rejoindre, à partir du paysage, les échelles du texte. Elle plaide en faveur d'une approche scalaire de la question du paysage en littérature. Le triangle du paysage littéraire a pour sommets le moi, le monde et les mots; il paraît évident que chaque genre, chaque courant, chaque auteur est conduit à privilégier l'un ou l'autre de ces pôles: la poésie lyrique met l'accent sur la dimension subjective du paysage, le naturalisme cherche à donner une description objective du monde extérieur, le formalisme donne l'initiative aux mots pour construire un espace essentiellement textuel. Cela ne signifie pas que, dans chacun de ces cas, les deux autres composantes du paysage soient purement et simplement absentes, mais leur importance est relativement moindre. Ces proportions variables pourraient être reportées sur une échelle qui ferait apparaître une gradation du plus objectif au plus subjectif ou au plus formel.

L'étude du paysage littéraire doit faire appel à un autre type d'échelle: celle des contextes dans lesquels il s'insère. Je reprendrai à ce propos l'exemple de Rimbaud, que j'ai étudié dans ma thèse. A la fin de

40. Voir M. Collot, L'horizon fabuleux. 
la quatrième section de l'illumination Enfance, l'horizon vers lequel se dirige «le petit valet suivant l'allée dont le front touche le ciel» semble coïncider avec «la fin du monde». On ne peut le comprendre qu'en resituant cette image dans une série de contextes emboîtés. Le plus large, c'est l'histoire de la langue, des idées, de la littérature et de la culture: il nous révèle que la valeur positive de l'horizon, porteur de tous les espoirs du romantisme en un avenir radieux placé sous le signe de l'Idéal ou de l'Au-delà, s'est soudain inversée au tournant du XIX ${ }^{e}$ siècle avec la crise des valeurs romantiques, qui laissent le ciel vide et l'avenir obscur. Mais ces connotations culturelles du motif s'accompagnent de connotations plus personnelles, qui apparaissent dans le contexte plus restreint de l'œuvre de Rimbaud, où apparaît avec insistance un véritable vertige de l'horizon, qu'un titre comme Enfance nous invite à mettre en rapport avec la vie de l'auteur. Ces résonances affectives se donnent à lire de façon encore plus précise dans le micro-contexte du poème, dont la composition fait apparaître l'éclatement du sujet entre des identités et des paysages multiples, auxquels il s'identifie aussi par le jeu des signifiants, comme le donne à entendre un syntagme tel que «le petit valet suivant l'allée dont le front touche le ciel», où l'ambiguïté syntaxique redouble la proximité phonique entre "allée» et "valet», sans qu'on puisse savoir auquel des deux attribuer ce «front» qui «touche le ciel».

Un tel parcours des échelles du texte et de ses contextes essaie de rendre justice à la polysémie et à la polyvalence de tout paysage littéraire, qui condense toujours en lui plusieurs niveaux de sens et donc de lecture. Mais la littérature n'est qu'une approche parmi d'autres d'une expérience étonnamment riche, qui revêt une dimension véritablement anthropologique puisqu'elle concerne toutes les dimensions de l'activité et de la culture humaines, depuis les plus concrètes jusqu'aux plus abstraites, de l'agriculture à la philosophie en passant par la randonnée en plaine ou en montagne. On ne peut bien comprendre ses expressions littéraires et artistiques sans prendre en compte l'apport des sciences de la nature, de l'homme et de la société. C'est à mes yeux l'un des grands mérites de Claude Reichler que de nous avoir appris à le faire. Le paysage a été pour lui comme pour moi un point de passage privilégié entre les disciplines, et entre nous un lieu de rencontre que j'espère avoir encore d'autres occasions de fréquenter avec lui. Pourquoi pas en nous promenant, comme il m’avait si gentiment invité à le faire du côté d'Aigle, 
il y a quelques années, dans le cadre d'une manifestation qui restera à jamais inscrite dans ma mémoire: son titre s'affichait jusque sur les panneaux de signalisation routière, si bien qu'en avançant on entrait dans un territoire de rêve, et pourtant bien réel, baptisé: «Paysages en poésie » ${ }^{41}$.

Michel Collot

Université Sorbonne Nouvelle-Paris III

4I. Voir P. Starobinski (dir.), Paysages en poésie. 


\section{BIBLIOGRAPHIE}

Backhaus, Norman, Reichler, Claude, Stremlow, Matthias, Paysages des Alpes. De la représentation à l'action, Zurich, VDF Editions de la Haute Ecole, 2007.

Bailly, Antoine, Scariati, Robert (dir.), L’humanisme en géographie, Paris, Anthropos, 1990.

Bergé, Aline, "Le tournant paysager de la littérature contemporaine", in Paysage et modernité(s), éd. par Aline Bergé, Michel Collot, Bruxelles, Ousia, 2007, p. 87-101.

Bouloumié, Arlette, Trivisani-Moreau, Isabelle (dir.), Le génie du lieu. Des paysages en littérature, Paris, Imago, 2005.

Bouvier, Nicolas, L'usage du monde, Paris, La Découverte, 1985.

Brosseau, Marc, Des romans-géographes, Paris, L'Harmattan, 1996.

Butor, Michel, Le génie du lieu, t. 1, Paris, Grasset, 1958.

—, Le génie du lieu, t. 2: Où, Paris, Gallimard, 1971.

—, Le génie du lieu, t. 3: Boomerang, Paris, Gallimard, 1978.

—, Le génie du lieu, t. 4: Transit A / Transit B, Paris, Gallimard, 1992.

—, Le génie du lieu, t. 5: Gyroscope, Paris, Gallimard, 1996.

Collot, Michel, L'horizon fabuleux, Paris, Corti, 1988.

—, "Petit discours sur les méthodes", in La traversée des thèses, éd. par

D. Alexandre, M. Collot, J.-Y. Guérin, M. Murat, Paris, Presses Sorbonne Nouvelle, 2005, p. 35-42.

—, Paysage et poésie, Paris, Corti, 2005.

—, La pensée-paysage. Philosophie, arts, littérature, Arles, Actes Sud/ Versailles, ENSP, 2011.

Collot, Michel, Rodriguez, Antonio (dir.), Paysage et poésies francophones, Paris, Presses Sorbonne Nouvelle, 2005.

Coyault, Sylviane, La province en héritage: Pierre Michon, Pierre Bergounioux, Richard Millet, Genève, Droz, 2002.

Echenoz, Jean, Je m'en vais, Paris, Editions de Minuit, 1999.

«Ecrire le paysage», Revue des sciences humaines, 209 (1988).

Ferré, André, Géographie de Marcel Proust, Paris, Le Sagittaire, 1939.

—, Géographie littéraire, Paris, Le Sagittaire, 1946. 
FRANK, Joseph, "La forme spatiale dans la littérature européenne» (1945), traduction française dans Poétique, 10 (1976), p. 244-266. Frémont, Armand, La région, espace vécu, Paris, PUF, 1976.

Gendrat-Claudel, Aurélie, Le paysage, "fenêtre ouverte" sur le roman: le cas de l'Italie romantique, Paris, Presses de l'université ParisSorbonne, 2007.

Genette, Gérard, «Espace et langage», in Figures I, Paris, Seuil, 1966, p. 101-108.

Glissant, Edouard, Tout-monde, Paris, Gallimard, 1993.

—, Traité du Tout-monde, Paris, Gallimard, 1997.

Le Bris, Michel, Rouaud, Jean (dir.), Pour une littérature-monde, Paris, Gallimard, 2007.

Le Clézio, Jean-Marie Gustave, Le livre des fuites, Paris, Gallimard, 1969.

Lhoste, Pierre, "Je fuis l'Europe des esclaves", entretien avec J.-M. G. Le Clézio, Les Nouvelles littéraires, 2249 (29 octobre 1970), p. 1.

Proust, Marcel, Du côté de chez Swann, in A la recherche du temps perdu, tome I, Paris, Gallimard, 1987.

Reichler, Claude, "Frontières du monde et territorialité du sujet", in L'humanisme en géographie, éd. par Antoine Bailly, Renato Scariati, Paris, Anthropos, 1990, p. 123-134.

—, La découverte des Alpes et la question du paysage, Chêne-Bourg/Paris, Georg, 2002.

- (dir.), Le voyage dans les Alpes, Chêne-Bourg/Genève/Paris, Georg, 2003.

—, "Le marcheur romantique et la phénoménologie du chemin", in Marche et paysage. Les chemins de la géopoétique, éd. par Bertrand Lévy, Alexandre Gillet, Genève, Métropolis, 2007, p. 31-64.

—, «Paysage et expérience de l'espace dans L'usage du monde», Europe, 974-975 (2010), p. 135-147.

Ruskin, John, Ecrits sur les Alpes, éd. par Emma Sdegno, Claude Reichler, traduction d'André Hélard, Paris, Presses de l'université Paris-Sorbonne, 2013.

Sangirardi, Giuseppe (dir.), Le paysage dans la littérature italienne, Dijon, Editions universitaires de Dijon, 2006.

Starobinski, Pierre (dir.), Paysages en poésie, Gollion, Infolio, 2004. 
THÉvoz, Samuel, Un horizon infini. Explorateurs et voyageurs français au Tibet (1846-1912), Paris, Presses de l'université Paris-Sorbonne, 2010.

Tuan, Yi-Fu, Espace et lieu: la perspective de l'expérience, Gollion, Infolio, 2006 (1977). 
